\title{
Electric Double-layer Capacitance and Pseudocapacitance Contributions to the Oxidative Modification of Helical Carbon Nanofibers
}

\author{
Yong Gong ${ }^{1,2}$, Xian-Guang Zeng ${ }^{2}$, Tong Luo ${ }^{2}$, Zu-Yang Dai ${ }^{2}$, Yong-Zhong Jin ${ }^{2}$, Jian Chen ${ }^{2}$, Yong Yi ${ }^{1}$, \\ Tao Duan ${ }^{1}$, Yong-Jian Tang ${ }^{1 *}$ \\ ${ }^{1}$ State Key Laboratory of environment-friendly energy materials, Southwest University of Science and \\ Technology, Mianyang, 621010, China \\ ${ }^{2}$ Material Corrosion and Protection Key Laboratory of Sichuan province, Sichuan University of \\ Science and Engineering, Zigong, 643000, China \\ *E-mail: tangyongjian2000@ sina.com (Yong-Jian Tang)
}

doi: $10.20964 / 2020.08 .94$

Received: 17 April 2020 / Accepted: 8 June 2020 / Published: 10 July 2020

Surface functionalization with oxygen-containing functional groups of helical carbon nanofibres (HCNFs) is a simple and effective method to increase the capacitive performance of the HCNFs. However, the contribution of the electric double-layer capacitance (EDLC) and pseudocapacitance (PC) to the overall behaviour of supercapacitors has not yet been explored. $\mathrm{A} \mathrm{HNO}_{3}$ treatment and the modified Hummers method were used to increase the oxygen-containing functional groups and specific surface area of the HCNFs. The increase in the specific surface area enhanced the EDLC, while the oxygen-containing functional groups led to PC. As a result of the modified Hummers method, the HCNFs with a specific surface area of $78.82 \mathrm{~m}^{2} / \mathrm{g}$ and containing 28.03 at. $\%$ oxygen had a specific capacitance of $143.6 \mathrm{~F} / \mathrm{g}$ at $1 \mathrm{~A} / \mathrm{g}$, which is much higher than that of the pristine HCNFs $(10.7$ F/g). In addition, the contribution of the EDLC and PC were evaluated and are discussed.

Keywords: Helical carbon nanofibers, pseudocapacitance, electric double-layer capacitance, oxidative modification

\section{$\underline{\text { FULL TEXT }}$}

(C) 2020 The Authors. Published by ESG (www.electrochemsci.org). This article is an open access article distributed under the terms and conditions of the Creative Commons Attribution license (http://creativecommons.org/licenses/by/4.0/). 